\title{
Gene silencing in Huntington's disease
}

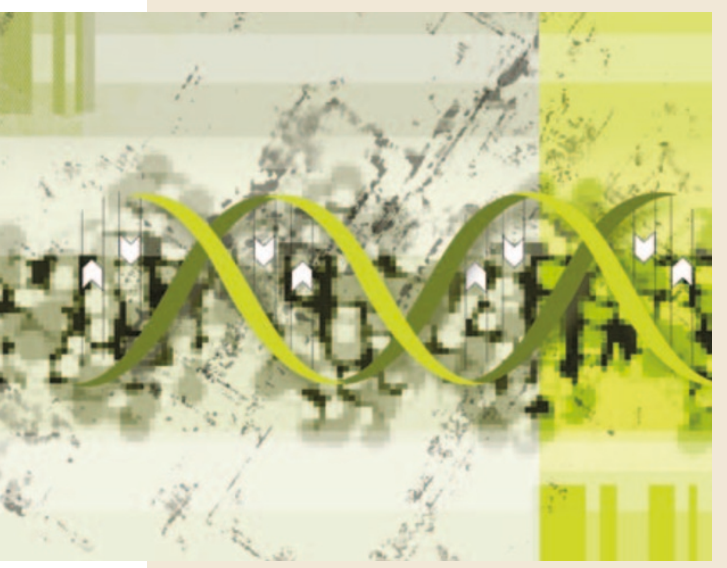

Huntington's disease is a dominantly inherited neurodegenerative disorder that results from expanded polyglutamine (polyQ) repeats in the huntingtin protein (HTT). This leads to progressive striatal and cortical neurodegeneration and associated motor, cognitive and behavioural disturbances. Reducing the expression of the mutant HTT gene using RNA interference

\section{Fly-by-light}

There has always been considerable interest in elucidating the roles of particular neurons and groups of neurons in behaviour, but it has been difficult to carry out such investigations on unrestrained animals. The recent use of biological 'labels' in particular neural populations has provided greater flexibility in this field, and research publised in Cell now reports the use of photostimulation to investigate the neural basis of movement in flies.

Lima and Miesenböck expressed optically gated ion channels in certain Drosophila melanogaster neurons and used pulses of unfocused laser light to allow them to link particular behaviours to specific populations of neurons. Furthermore, by using a mutation that blocks phototransduction, they were able to ensure that their results reflected the stimulation of target neurons and not simply activation of the visual system.

Escape movements - including jumping, wing beating and flight — were seen in a high
(RNAi) has emerged as a promising treatment for this fatal neurodegenerative disorder, according to a new report in the Proceedings of the National Academy of Sciences USA.

Previous attempts to develop therapeutic interventions for Huntington's disease have focused on targeting the downstream effectors of HTT expression. However, until now, there have been no efforts to directly target the underlying mutant gene.

Davidson and colleagues injected vectors that encode short hairpin RNA sequences into a transgenic mouse model of Huntington's disease. This resulted in reduced expression of mutant human HTT in the striatum and marked improvements in motor and neuropathological abnormalities. The authors suggest that the hairpin sequence could be further optimized according to more recently issued guidelines, which might lead to greater therapeutic benefit.

In this study, RNAi affected the expression of mutant human HTT but not normal mouse Htt owing to differences in the mouse and human genes. However, if this method were used in human patients, the expression of both the normal and the mutated copies of the gene would be reduced, which raises the interesting question of whether reduced expression of both normal and mutated HTT could be tolerated in humans. This question needs to be tackled before RNAi can be tested therapeutically in humans with Huntington's disease. Nevertheless, these results provide encouraging evidence that gene silencing techniques could be developed to directly target the underlying genetic abnormality in Huntington's disease, and, potentially, other neurodegenerative disorders.

Alison Rowan

\section{(1) References and links}

ORIGINAL RESEARCH PAPER Harper, S. Q. et al. RNA interference improves motor and neuropathological abnormalities in a Huntington's disease mouse model. Proc. Natl Acad. Sci. USA 5820-5825 (2005) FURTHER READING Beal, M. F. \& Ferrante, R. J. Experimental therapeutics in transgenic mouse models of Huntington's disease. Nature Rev. Neurosci. 5, 373-384 (2004) | Davidson, B. L. \& Breakefield, X. O. Neurological diseases: viral vectors for gene delivery to the nervous system. Nature Rev. Neurosci. 4, 353-364 (2003) percentage of flies in response to photostimulation of giant fibre neurons. However, flight and wing beating endured for considerably longer time periods than the stimulus, which, the authors suggest, indicates that giant fibre neurons simply act to set the thoracic oscillations that control wing movement in motion.

Dopaminergic neurons are also involved in movement control, and Lima and Miesenböck used this model to show that an increase in dopaminergic signalling leads to an increase in locomotor activity and variations in walking patterns. However, the speed at which the flies moved did not change, and the authors ascribe the increase in locomotion to shorter and less frequent pauses during walking.

The authors conclude that the expression of phototriggers in neurons not only opens the door for further research, but might also provide a means of treating diseases and injuries of the CNS in the future.

Sarah Archibald

(2) References and links

ORIGINAL RESEARCH PAPER Lima, S. Q. \& Miesenböck, G. Remote control of behavior through genetically targeted photostimulation of neurons. Cell 121, 141-152 (2005)

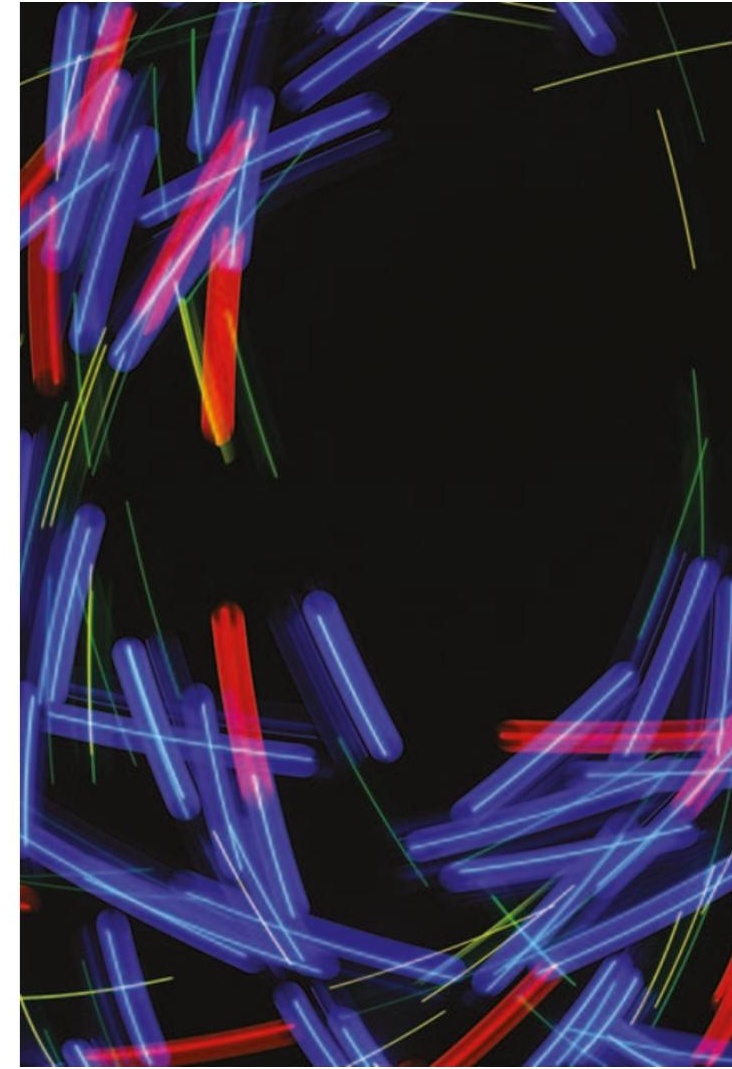

